\title{
Dynamic of Excited States of Spatially Separated Qubits in a Waveguide
}

\author{
E.S. Redchenko ${ }^{1,2}$ a and V.I. Yudson ${ }^{2,3}$ \\ ${ }^{1}$ Moscow Institute of Physics and Technology, Dolgoprudny, Russia \\ ${ }^{2}$ Institute of Spectroscopy, Russian Academy of Sciences, Troitsk, Russia \\ ${ }^{3}$ Russian Quantum Center, Skolkovo, Russia
}

The light-matter interaction effects are especially important in one-dimentional (1D) systems, where there is no spatial spreading of emitted radiation. We study [1] formation and decay of metastable (dark) states in a system of two spatially separated qubits (two-level atoms) coupled to a 1D waveguide. Such metastable states of a singly excited pair of resonant atoms arise when the distance between the atoms takes special values [2]. We have calculated their decay rate when the interatomic distance deviates from the special values or when the transition frequencies of the two atoms do not coincide (inhomogeneous detuning). Also, we have found that the doubly excited state of two resonant atoms located at the special positions does not completely decay, but with a finite probability can evolve to one of the metastable singly-excited states. Dark states of pairs of qubits may serve as memory elements or sensitive detectors of external perturbations.

\section{References}

1. E.S. Redchenko and V.I. Yudson, Phys. Rev. A 90, 063829 (2014)

2. A.A. Makarov and V.S. Letokhov, JETP 97, 688 (2003)

\footnotetext{
${ }^{\mathrm{a}}$ Corresponding author: lenaredchenk@rambler.ru
} 